\title{
The Clinical and Radiological Outcome of Cervical Spinal Meningioma
}

\author{
Yong-Jun Lee, Jong-myung Jung, Seung-Jae Hyun, Ki-Jeong Kim, Tae-Ahn Jahng, Hyun-Jib Kim \\ Department of Neurosurgery, Spine Center, Seoul National University Bundang Hospital, Seoul National University \\ College of Medicine, Seongnam, Republic of Korea
}

Corresponding author:

Jong-myung Jung

Department of Neurosurgery, Spine Center, Seoul National University

Bundang Hospital, Seoul National University College of Medicine, 82,

Gumi-ro, 173 beon-gil, Bundang-gu, Seongnam 13620, Republic of Korea

Tel: +82-31-787-6293

Fax: +82-31-787-4097

E-mail: beast2625@snu.ac.kr

Received: August 3, 2018

Revised: September 3, 2018

Accepted: September 6, 2018

\begin{abstract}
Objective: We aimed to present the clinical and radiological outcomes of 22 cases of cervical spinal meningiomas. Methods: We retrospectively reviewed 22 patients who were diagnosed with cervical meningioma histologically from May 2003 to May 2017. Their pre- and postoperative clinical features were evaluated by the Japanese Orthopaedic Association (JOA) scale and Neck Disability Index (NDI). Results: There were 6 male and 16 female patients. The mean age was 60 years (20-73 years). The most common preoperative symptom was paresthesia in 15 patients. The craniocaudal tumor extension of less than 3 levels was found in 18 patients. More than $50 \%$ of spinal canal compression was observed in 16 patients. Most common location of the dural attachment of the tumor was ventrolateral $(54.5 \%)$, followed by dorsolateral (18.2\%), lateral (13.6\%), ventral (9.1\%) and dorsal (4.5\%) order. Extradural tumor extension was observed in 5 patients. Simpson grade II resection was the most common, with 16 patients. The mean JOA scales were significantly improved from $13.8 \pm 2.6$ to $15.9 \pm 1.0$ at postoperative one year $(p=0.0004)$. The mean NDI showed the same results (from $14.6 \pm 8.2$ to $8.4 \pm 6.6$; $p=0.0005$ ). One patient with Simpson grade IV resection received adjuvant radiotherapy. Two patients had a recurrence of the tumor, one with Simpson grade II resection and the other with Simpson grade III resection. Conclusion: The surgery is the recommended treatment in cases of cervical spinal meningiomas because of its excellent functional improvement and low recurrence rates.
\end{abstract}

Key Words: Cervical vertebrae; Meningioma; Recurrence; Spinal neoplasms

\section{INTRODUCTION}

Spinal meningiomas are typically slow-growing, benign, and well-circumscribed neoplasms which located in the intraduralextramedullary space that account for $25 \%$ to $46 \%$ of all primary spinal tumors ${ }^{2,4,9,13)}$. The highest incidence of spinal meningio$\mathrm{ma}$ is in women in their fifth to seventh decade due to the frequent presence of estrogen receptors ${ }^{14)}$. They arise predominantly in the thoracic spine but can be found at any level of the spine ${ }^{8)}$. Whereas cervical spinal meningiomas are comparatively small portions of spinal meningiomas ${ }^{1,5,11}$, the surgical outcomes of cervical spinal meningiomas have rarely been described in previous literature. The purpose of the present study is to report the outcome of 22 patients with cervical spinal meningiomas.

\section{MATERIALS AND METHODS}

From the dataset of 34 patients who were consecutively operated at our institution between May 2003 and May 2017 for cervical spinal meningiomas. Five patients with craniocervical meningiomas with intracranial extension and foramen magnum were excluded. Seven patients with duration of followup of less than one year after surgery were excluded. Finally, 22 patients were included in this study.

\section{Clinical and Radiologic Evaluations}

Medical records including primary preoperative symptom, duration of symptom, postoperative complications, and adjuvant therapy were reviewed. Japanese Orthopaedic Association (JOA) scale and Neck Disability Index (NDI) were measured preoperatively and one year after surgery. Craniocaudal extension, degrees of spinal cord compression, the location of dural attachment, and extradural tumor extension were reviewed by preoperative magnetic resonance imaging (MRI). Surgical approach and extent of resection (according to Simpson grade) were inferred from the operative reports and confirmed with postoperative MRI. Recurrence after tumor removal was defined as MRI 
evidence of new tumor, as per the radiologist's report ${ }^{12)}$.

All surgeries were aimed at gross total resection. Whenever necessary, a surgical aspirator (cavitron ultrasonic surgical aspirator) was also applied to avoid any surgical traction on the cord. Generally, delicate and cautious cauterization of the dural attachment was performed instead of dural excision.

\section{Statistical Analysis}

The statistical analyses were performed using the Wilcoxon Signed Rank test or paired t-test for continuous variables between the pre- and postoperative neurological status according to the JOA scale and NDI. JOA scale was statistically nonparametric and Wilcoxon signed-rank test was performed. NDI showed parametric distribution of normality test and paired t-test was used. A two-sided p-value of $<0.05$ was considered significant. Statistical procedures were performed using a commercially available software package (SAS version 9.4; SAS Institute Inc., Cary, NC, USA).

\section{RESULTS}

The patients consisted of 16 women and 6 men (Table 1 ). The mean age was $60.0 \pm 13.0$ years (range, 20-73 years). The mean duration of symptoms until surgery was $11.2 \pm 20.5$ months. The chief complaints were paresthesia (15 patients, 68.2\%), motor weakness (10 patients, $45.5 \%)$, and gait disturbance (1 patient, $4.5 \%)$. The mean duration of follow-up after surgery was $40.0 \pm 26.7$ months.

Tumors were limited to 1 and 2 levels in 18 patients (81.8\%), but 16 patients $(72.7 \%)$ had spinal canal compression ratio of more than 50\% (Table 2). Preoperative computed tomography (CT) scans were performed in 13 of 22 patients, of whom 6 had calcification.

The dural attachment of the cervical spinal meningioma was predominantly localized ventrolaterally in $12(54.5 \%)$ patients

Table 1. Demographic data in cervical meningioma patients

\begin{tabular}{lc}
\hline \hline Variables & No. of patients (\%) $(\mathrm{n}=22)$ \\
\hline Sex & $16(72.7)$ \\
Female & $6(27.3)$ \\
Male & $60.0 \pm 13.0$ \\
Age (years) & $11.2 \pm 20.5$ \\
Duration of symptoms (months) & \\
Preoperative chief complaint & $15(68.2)$ \\
Paresthesia & $10(45.5)$ \\
Motor weakness & $1(4.5)$ \\
Gait disturbance & $40.0 \pm 26.7$ \\
Mean follow-up (months) &
\end{tabular}

The data is presented as number (\%) or mean \pm standard deviation.
(Fig. 1). Four meningiomas were located in the dorsolateral, three in the lateral, two in the ventral, and one in the dorsal. Extradural tumor extension was observed in 5 patients, and all of these tumors were extended to neural foramen.

Twenty-one patients (95.5\%) underwent standard posterior laminectomies (Table 3). The combined anterior and posterior approach was performed in only 1 patient at an interval of 4 days. Simpson grade I resection was achieved in 3 patients (13.6\%), grade II in $16(72.7 \%)$, grade III in $1(4.5 \%)$ and grade IV in 2 patient (9.1\%). Histological types were as follows: 14 meningotheliomatous, four psammomatous, one transitional, one microcystic, one metaplastic, and one fibrous.

The functional improvement after surgery showed statistical

Table 2. Imaging characteristics in cervical meningioma patients

\begin{tabular}{lc}
\hline \hline Variables & $\begin{array}{c}\text { No. of patients }(\%) \\
(\mathrm{n}=22)\end{array}$ \\
\hline Craniocaudal tumor extension & $18(81.8)$ \\
$1-2$ levels & $4(18.2)$ \\
$\geq 3$ levels & \\
The extent of spinal cord compression & $16(72.7)$ \\
Tumor extension $>50 \%$ of spinal canal & $6(27.3)$ \\
Tumor extension $<50 \%$ of spinal canal & \\
Calcification & $7(31.8)$ \\
None & $6(27.3)$ \\
Calcified & $9(40.9)$ \\
Not identified &
\end{tabular}

The data is presented as number (\%).

Table 3. Surgical characteristics and histological type in cervical meningioma patients

\begin{tabular}{lc}
\hline \hline Variables & No. of patients (\%) $(\mathrm{n}=22)$ \\
\hline Surgical approach & $0(0.0)$ \\
Anterior & $21(95.5)$ \\
Posterior & $1(4.5)$ \\
Combined (anterior+posterior) & \\
Simpson resection grade & $3(13.6)$ \\
I & $16(72.7)$ \\
II & $1(4.5)$ \\
III & $2(9.1)$ \\
IV & \\
Histological grade \& type & $14(63.6)$ \\
Meningotheliomatous & $4(18.2)$ \\
Psammomatous & $1(4.5)$ \\
Transitional & $1(4.5)$ \\
Microcystic & $1(4.5)$ \\
Metaplastic & $1(4.5)$ \\
Fibrous &
\end{tabular}




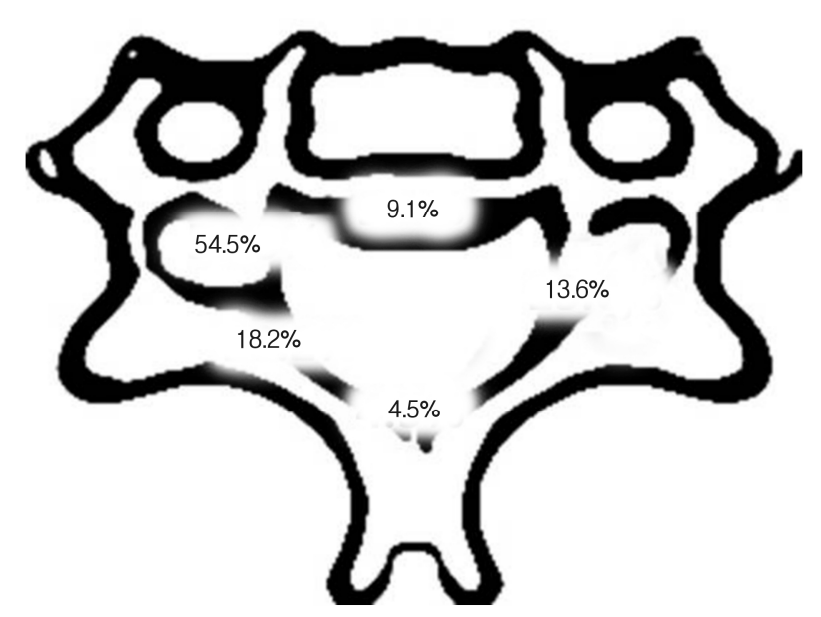

Fig. 1. Localization of the dural attachment of the spinal meningioma. significance (Fig. 2). The mean JOA scale was improved from $13.8 \pm 2.6$ to $15.9 \pm 1.0(\mathrm{p}<0.001)$ at postoperative one year. The NDI was also improved from $14.6 \pm 8.2$ to $8.4 \pm 6.6$ ( $\mathrm{p}<0.001)$.

There were three surgical related complication. One patient had a cerebrospinal fluid leakage; however, conservative treatment was performed without reoperation. Other 2 patients had monoparesis after surgery. One patient received additional radiation therapy. The overall recurrence rate in this series was 9.1\% (2 patients), each patient received Simpson grade II and Simpson grade III resection. Despite recurred tumor, this group of patients fared well without additional therapy.

\section{Illustrative Case}

A 55-year-old woman presented with posterior neck pain and both shoulder tingling sense for 12 months. MRI of the

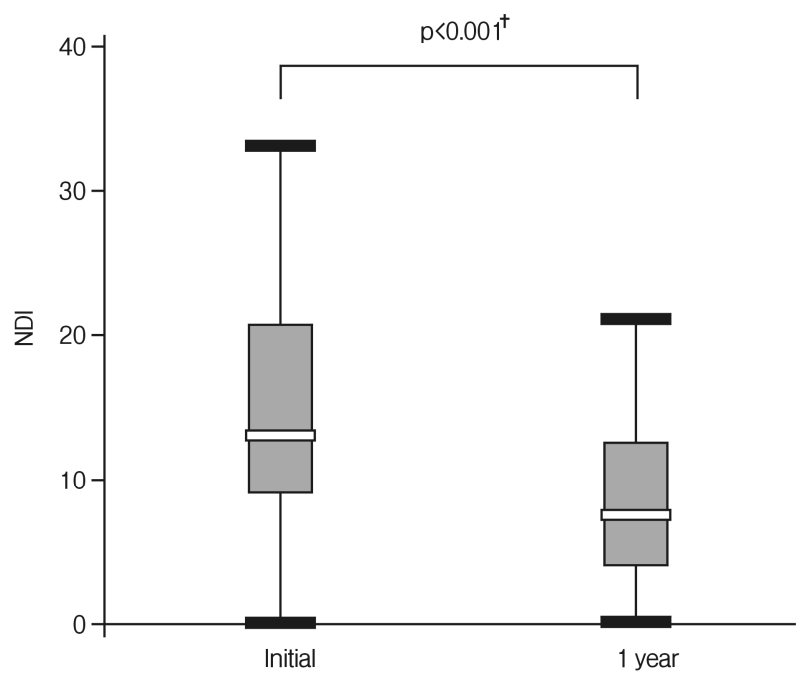

Fig. 2. Pre- and postoperative neurological status according to the Japanese Orthopedic Association (JOA) score and Neck Disability Index (NDI). *Wilcoxon Signed Rank test, $p=0.0004,{ }^{+}$Paired t-test, $p=0.0005$.
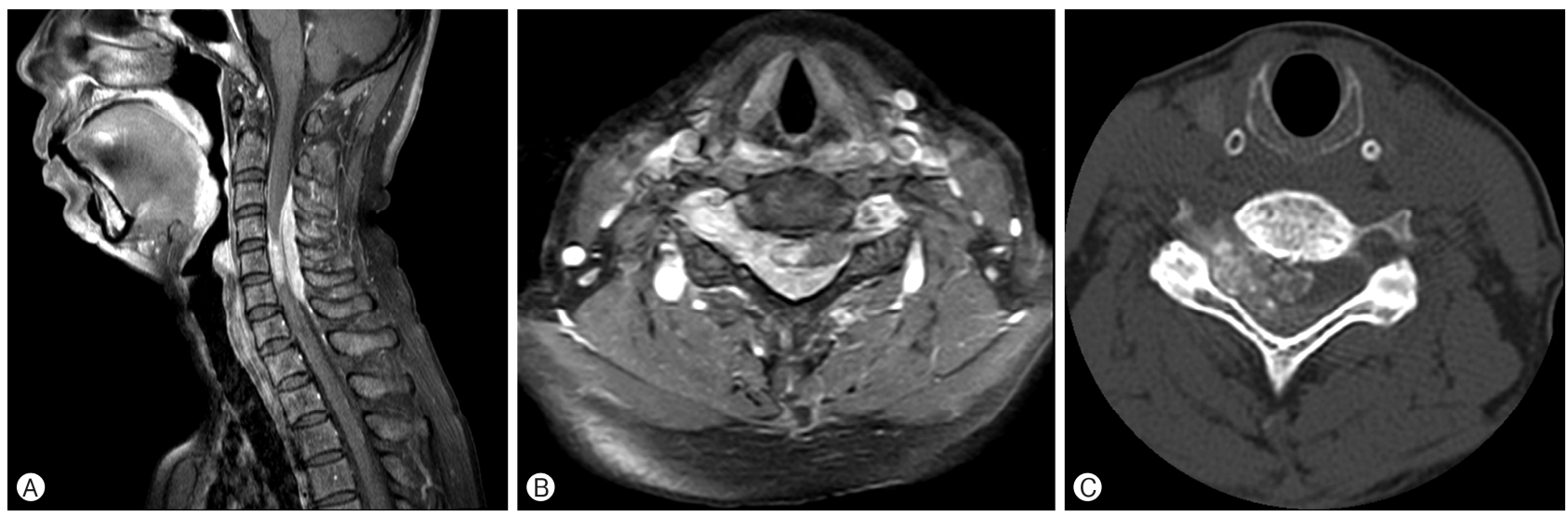

Fig. 3. (A) Illustrative case. Preoperative gadolinium enhanced T1-weighted sagittal magnetic resonance image showing an extensive en plaque shaped spinal meningioma with dural attachment at the level C3 to C6. (B) Illustrative case. Preoperative gadolinium enhanced T1weighted axial magnetic resonance image revealing tumor on the right side of the spinal canal and C5/6 neural foramina, with significant spinal cord compression. (C) Illustrative case. Preoperative computerized tomography showing neuroforaminal widening at right $\mathrm{C} 5 / 6$ by calcified mass. 
cervical spine revealed an en plaque shape enhancing extradural mass at C3-6 level (Fig. 3A) on the right side of the spinal canal and C5-6 neural foramina, with significant spinal cord compression, more than 50\% (Fig. 3B). CT of the cervical spine showed widening of the right-side C5-6 neural foramina by calcified mass (Fig. 3C).

On physical examination, the patient had full muscle strength except posterior neck pain and paresthesia on both shoulder.

A C3-6 laminectomy and subtotal resection of tumor were performed. Due to proximity to the vertebral artery, only subtotal resection (Simpson grade IV) was attempted. In the operative view, the tumor had severe adhesion to spinal cord. The pathologic review of the tumor showed a psammomatous meningioma. Immediately after surgery, the patient manifested weakness of the left deltoid (3/5), biceps (4/5), hand grip (4/5), which progressively returned to motor grade $4+$ with continuous rehabilitation.

The patient underwent adjuvant radiotherapy 10 months after surgery to the residual lesion in the right epidural space involving neural foramina of C5-6 due to deficient spinal cord decompression.

The patient was followed by using serial MRI at 10, 30, 42, 60, and 96 months after operation and images showed no remarkable interval change of residual tumor.

\section{DISCUSSION}

Although many kinds of literature report the surgical outcome of spinal meningiomas, there were few cohort studies which separately featuring the cervical spinal meningiomas. It may be due to relatively a small proportion of cervical origin compared with other origins. This study presents characteristics of cervical spinal meningioma with surgical outcomes.

The most common presenting symptoms were paresthesia (15 patients, 68.2\%) in our series and the mean duration of symptoms before presentation was $11.2 \pm 20.5$ months. Majority of prior reports of spinal meningiomas ${ }^{3,5,10,15)}$ informed similar predominant symptom and duration. Also, prior studies reported $26.3 \%$ to $51 \%$ of bladder and sphincter disturbances, but our series showed no significant difference in bladder and sphincter disturbances. The reason of this obvious distinction is that our study was implied to cervical location. The most common axial location in our series was ventrolateral (12 patients, 54.5\%); dorsolateral location was represented in 4 patients (18.2\%). As previously reported by other authors, the ventral/ventrolateral topography is a quite common location for the dural attachment of the spinal meningiomas (38.5-65.2\%) $)^{3,9)}$. The meningothelial type (14 patients, $63.7 \%$ ) of meningioma was the most common histology subtype in our series, followed by psammomatous in 4 patients (18.2\%). Maiti et al." found no correlations between histological subtype and functional outcome. However, Schaller ${ }^{10)}$ had a different opinion that the presence of a psammoma- tous meningioma has been reported as being predictive of postoperative deterioration as these tumors are more adherent to the spinal cord compared to other histological subtypes. In our series, there was 1 patient whom aforementioned in illustrative case had psammomatous meningioma coincided with this theory.

The functional outcome improvement rate after surgery in this study was $86.4 \%$. The JOA score and NDI were significantly improved. Our surgical outcome of functional status was similar to other literature, which showed $71.3 \%$ to $92 \%$ of patients with postoperative functional improvement ${ }^{4,5,7,11,15)}$.

Two cases (9.1\%) of attempted subtotal resection (Simpson grade IV) had several common features such as the extension of more than two levels in the craniocaudal direction, severe adhesion to the spinal cord, and presence of calcifications. One of these patients was presented in the illustrative case above and underwent adjuvant radiotherapy ten months after surgery.

The overall recurrence rate in this series was 9.1\% (2 patients), each patient received Simpson grade II (1/16) and Simpson grade III (1/1) resection. Two patients who had Simpson grade IV resection had no recur, which is significantly low than the other literature which reported $87.5 \%$ of recurrence in Simpson grade IV removal $^{6}$.

The main limitations of this study are limited numbers, its retrospective nature, and relatively short follow-up times. Moreover, there was no malignant meningioma in our cases. The reported recurrence-free in Simpson grade IV resection could be falsely high in this series if a clinically silent recurrence develops before it is diagnosed on imaging. Multiple reports proposed that World Health Organization grade II or III meningiomas are relatively prone to recurrence. Despite these significant limitations, this study contributes to the scant literature on cervical spinal meningiomas that thus further study can be published later on.

\section{CONCLUSION}

Based on the outcomes of this study, we conclude that surgery for cervical spinal meningioma has a favorable clinical outcome in the majority of patients at least for the first year after the operation.

\section{CONFLICT OF INTEREST}

No potential conflict of interest relevant to this article was reported.

\section{REFERENCES}

1. Abul-Kasim K, Thurnher MM, McKeever P, Sundgren PC: Intra- 
dural spinal tumors: current classification and MRI features. Neuroradiology 50:301-314, 2008

2. Ambekar S, Sharma M, Kukreja S, Nanda A: Complications and outcomes of surgery for spinal meningioma: a Nationwide Inpatient Sample analysis from 2003 to 2010. Clin Neurol Neurosurg 118:65-68, 2014

3. Frati A, Pesce A, Toccaceli G, Fraschetti F, Caruso R, Raco A: Spinal meningiomas prognostic evaluation Score (SPES): predicting the neurological outcomes in spinal meningioma surgery. Neurosurg Rev [epub ahead of print Mar 7, 2018. doi: 10.1007/ s10143-018-0961-1]

4. Gezen F, Kahraman S, Canakci Z, Beduk A: Review of 36 cases of spinal cord meningioma. Spine (Phila Pa 1976) 25:727-731, 2000

5. Gottfried ON, Gluf W, Quinones-Hinojosa A, Kan P, Schmidt MH: Spinal meningiomas: surgical management and outcome. Neurosurg Focus 14:e2, 2003

6. Kim $\mathrm{CH}$, Chung CK, Lee SH, Jahng TA, Hyun SJ, Kim KJ, et al.: Long-term recurrence rates after the removal of spinal meningiomas in relation to Simpson grades. Eur Spine J 25:40254032, 2016

7. Maiti TK, Bir SC, Patra DP, Kalakoti P, Guthikonda B, Nanda A: Spinal meningiomas: clinicoradiological factors predicting recurrence and functional outcome. Neurosurg Focus 41:E6, 2016
8. Ottenhausen M, Ntoulias G, Bodhinayake I, Ruppert FH, Schreiber S, Förschler A, et al.: Intradural spinal tumors in adults-update on management and outcome. Neurosurg Rev [epub ahead of print, 2018. doi: 10.1007/s10143-018-0957-x]

9. Sandalcioglu IE, Hunold A, Muller O, Bassiouni H, Stolke D, Asgari S: Spinal meningiomas: critical review of 131 surgically treated patients. Eur Spine J 17:1035-1041, 2008

10. Schaller B: Spinal meningioma: relationship between histological subtypes and surgical outcome? J Neurooncol 75:157-161, 2005

11. Setzer M, Vatter H, Marquardt G, Seifert V, Vrionis FD: Management of spinal meningiomas: surgical results and a review of the literature. Neurosurg Focus 23:E14, 2007

12. Simpson D: The recurrence of intracranial meningiomas after surgical treatment. J Neurol Neurosurg Psychiatry 20:22-39, 1957

13. Solero CL, Fornari M, Giombini S, Lasio G, Oliveri G, Cimino, et al.: Spinal meningiomas: review of 174 operated cases. Neurosurgery 25:153-160, 1989

14. Westwick HJ, Shamji MF: Effects of sex on the incidence and prognosis of spinal meningiomas: a Surveillance, Epidemiology, and End Results study. J Neurosurg Spine 23:368-373, 2015

15. Yoon SH, Chung CK, Jahng TA: Surgical outcome of spinal canal meningiomas. J Korean Neurosurg Soc 42:300-304, 2007 